\title{
Ubiquitylation and the pathogenesis of hypertension
}

\author{
David H. Ellison'1,2
}

1Division of Nephrology and Hypertension, Department of Medicine, Department of Physiology and Pharmacology, Heart Research Center, Oregon Health and Science University, Portland, Oregon, USA. ${ }^{2}$ VA Medical Center, Portland, Oregon, USA.

\author{
Because something is happening here \\ But you don't know what it is \\ Do you, Mister Jones? \\ -Bob Dylan, \\ "Ballad of A Thin Man” (1965)
}

Liddle syndrome is monogenic hypertension caused by mutations in the epithelial $\mathrm{Na}^{+}$channel (ENaC) that interfere with its ubiquitylation by Nedd4-2. In this issue, Ronzaud and colleagues found that deleting Nedd4-2 from kidney tubules in adult mice led to $\mathrm{ENaC}$ accumulation, but not at the plasma membrane, as predicted from current models. Instead, abundance of the sodium chloride transporter NCC increased at the plasma membrane, and the mice have some features of increased NCC activity. Together, the results suggest that defective ubiquitylation of ENaC by Nedd4-2 may not fully explain Liddle syndrome and that Nedd4-2 modulates NCC more strongly.

Liddle syndrome is a monogenic hypertensive disease that results from excess activity of the epithelial sodium channel ENaC (1). In 1996, Staub, Rotin, and colleagues found that the ubiquitin ligase Nedd 4-2 interacts with $\mathrm{ENaC}$ via PY motifs within the carboxyl terminal domains of $\mathrm{ENaC}$ subunits (1). The disease-causing mutations in the $\beta$ - or $\gamma$ ENAC subunits result in deletion of the PY motifs (2), which abrogates ubiquitylation of the channel in vitro (3). As ubiquitylation of proteins appears to accelerate their removal from the plasma membrane and to increase degradation (or recycling), these deletions are expected to result in an increase in $\mathrm{ENaC}$ at the plasma membrane, an effect that has been observed both in vitro (3) and in vivo (4); mice with a mutated $\beta E N a C$ also exhibit many of the phenotypic features of Liddle syndrome, supporting the fidelity of this model (4).

Although attention has focused on the ability of Nedd4-2 to modulate ENaC, it may also interact with another sodium transport protein expressed by the distal nephron, the thiazide-sensitive $\mathrm{Na}^{+} \mathrm{Cl}^{-}$ cotransporter (NCC, SLC12A3). Ko and colleagues reported that NCC is ubiquitylat-

Conflict of interest: The author has declared that no conflict of interest exists.

Citation for this article: J Clin Invest. 2013; 123(2):546-548. doi:10.1172/JCI66882. ed, and suggested that this process might be involved in its endocytosis (5). Arroyo et al. reported that NCC coimmunoprecipitates with Nedd4-2 when transfected into HEK293 cells and that coexpression of Nedd4-2 with NCC inhibited NCC activity in oocytes (6). Surprisingly, however, the interaction of Nedd4-2 with NCC did not occur through classical PY motifs, suggesting that the interaction occurs via alternative domains. Thus, emerging evidence posits a role for Nedd4-2 in mediating NCC ubiquitylation and endocytosis.

Nedd4-2 ubiquitin ligase activity is not constitutive, but instead is believed to be important in modulating aldosterone effects on ENaC and perhaps NCC. According to consensus models (Figure 1), aldosterone increases SGK1 expression, which phosphorylates Nedd4-2 on key serine residues, thereby promoting interaction with 14-3-3 proteins. This interaction reduces Nedd4-2's ubiquitin ligase activity with respect to $\mathrm{ENaC}$. The model suggests that $\mathrm{ENaC}$ that is not highly ubiquitylated will remain in the plasma membrane longer (7). This hypothesis is supported by abundant data, but like all models, it must be tested in vivo to confirm its essential features. The paper by Ronzaud and colleagues (8) in this issue of the JCI takes this model out for a test drive, with some very surprising results.

\section{Nedd4-2 regulation of $\mathrm{ENaC}$}

Ronzaud et al. used an inducible tissuespecific recombination strategy to delete Nedd4-2 in the adult nephron (8), a system that circumvents the confounding effects of the gene's developmental and extrarenal roles (9). As expected, Nedd4-2 deletion led to substantial increases in $\beta$ - and $\gamma \mathrm{ENaC}$ abundance. What was unexpected was that the increase was predominantly inside the cell, rather than at the plasma membrane, and that $\alpha \mathrm{NaC}$ levels were substantially reduced. Although plasma aldosterone was reduced, suggesting positive sodium balance, the mice did not retain $\mathrm{Na}$ abnormally when switched to a high-salt diet, and serum potassium balance remained normal (8); thus, the mouse model does not phenocopy Liddle syndrome. These are striking and confusing results, suggesting on their surface that the consensus model for $\mathrm{ENaC}$ regulation by Nedd4-2-mediated ubiquitylation is either incorrect or incomplete. How can they be explained?

A prior study found that arterial pressure and $\mathrm{ENaC}$ abundance (including $\alpha \mathrm{ENaC}$ ) were elevated, as expected, when Nedd4-2 was deleted globally (10), but those investigators did not determine whether increased $\mathrm{ENaC}$ was located at the plasma membrane or inside the cell. Importantly, aldosterone levels were not suppressed in the global Nedd4-2-knockout animals, in striking contrast with the results obtained by Ronzaud and colleagues and with the findings in individuals with Liddle syndrome. This suggested to the prior investigators (10) that Nedd4-2 plays a role in modulating adrenal aldosterone secretion. As in the nephron-specific deletion model, global deletion of Nedd4-2 did not affect plasma potassium, again differing from the Liddle syndrome phenotype. Ronzaud et al. made an attempt to determine whether the differences between the global Nedd4-2 deletion model and the inducible kidney-specific model resulted from developmental or systemic factors by treating pregnant animals with doxycycline to delete Nedd4-2 in the kidney embryonically. The resulting phenotype, including the suppressed aldosterone, was essentially identical to that observed when Nedd4-2 was deleted in the kidney in adulthood (9). Taken together, these results suggest the provocative hypothesis that 
A

Extracellular

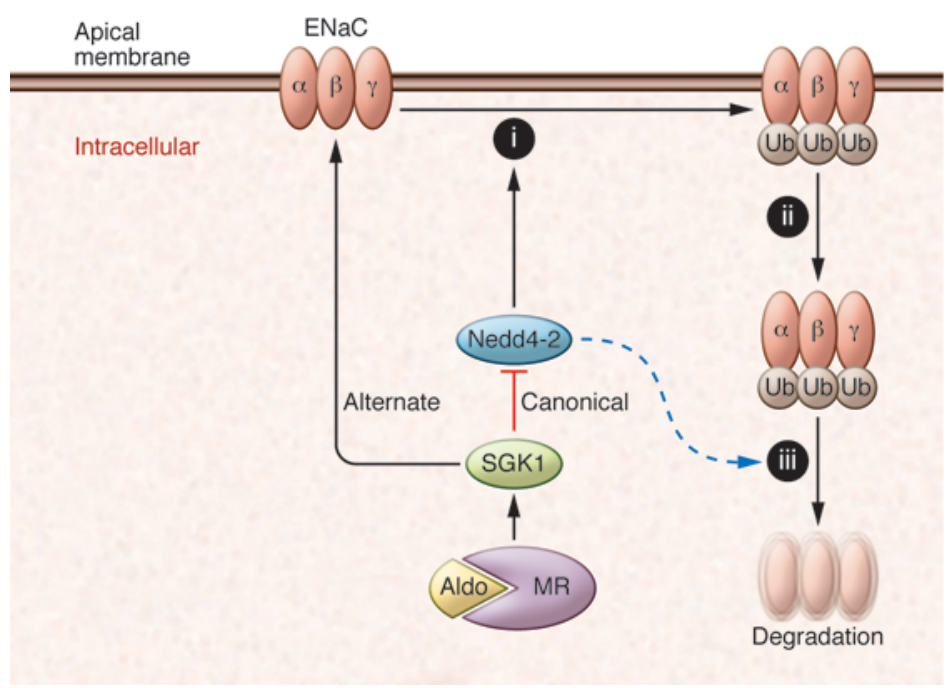

B

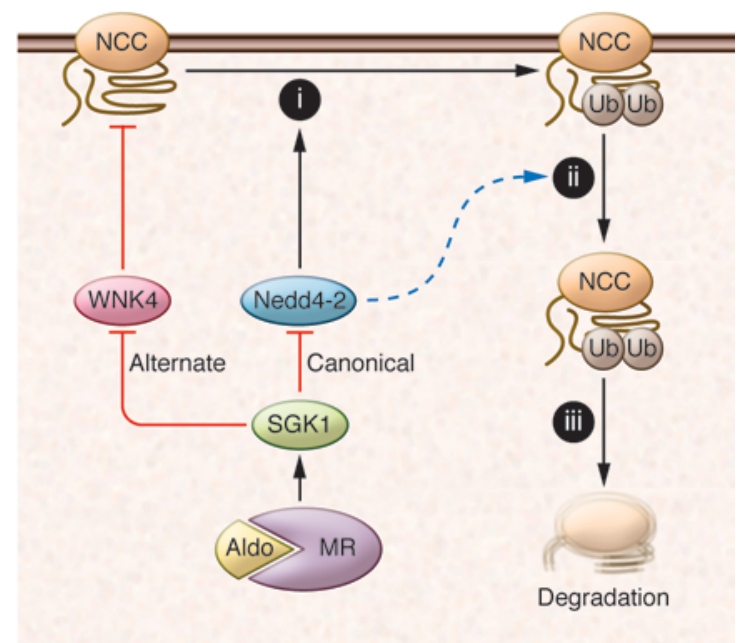

\section{Figure 1}

Models of ENaC and NCC regulation by Nedd4-2. (A) Aldosterone (Aldo) interaction with the mineralocorticoid receptor (MR) induces SGK1 activity, which suppresses the ubiquitin ligase activity of Nedd4-2. Nedd4-2 normally catalyzes the addition of ubiquitin moieties (Ub) to ENaC (step i). Ubiquitylated ENaC is then removed from the plasma membrane (step ii) and undergoes degradation (or recycling) inside the cell (step iii). Poorly defined alternate pathways for SGK1 to stimulate ENaC independently of Nedd4-2 also exist. Ronzaud and colleagues (8) found that Nedd4-2 deletion increased in intracellular ENaC, suggesting that Nedd4-2 may affect step iii (dotted blue arrow) more than step ii. (B) Similar signaling pathway to NCC. An alternative pathway for SGK1 activation of NCC may involve WNK4, as described in text. In contrast with effects on ENaC, NCC abundance was increased at the plasma membrane by Nedd4-2 deletion, suggesting a defect at step ii, as shown by the dotted blue arrow. Note that sites of ubiquitylation on NCC have not been defined.

the major effect of Nedd4-2 on $\mathrm{ENaC}$ is to modulate intracellular degradation (step iii in Figure 1A) rather than removal from the plasma membrane (step ii in Figure 1A); the abundance of $\alpha \mathrm{ENaC}$ is then likely regulated primarily by aldosterone.

One final possible explanation for the surprising results should be noted. Although both the nephron-specific (8) and global Nedd4-2-knockout (10) mice are viable and appear healthy, a third model of Nedd4-2knockout exhibited perinatal mortality due to pulmonary dysfunction (11). Both of the viable models inactivate Nedd4-2 by deleting exons $6-8$, while the mice with lethality had exons 15 and 16 deleted. Boase and colleagues (11) showed that deleting exons 6-8 results in exon skipping and the formation of truncated Nedd4-2 peptides and suggested that the residual function of these truncated forms abrogates perinatal lethality. Ronzaud et al. used several antibodies to provide evidence that production of such aberrant Nedd4-2 products does not occur, at least in the kidneys of their model (8). While these results are reassuring, an inducible nephron-specific Nedd4-2 model in which exons 15 and 16 are deleted should be generated to explore this difference.

\section{Nedd4-2 regulation of NCC}

The other notable result of the studies of Ronzaud and colleagues is that Nedd42 deletion led to a substantial increase in NCC abundance; this time, the increase appears to be primarily at the apical membrane, and the transporter appears to be active (8). This in vivo evidence that Nedd42 -dependent ubiquitylation modulates NCC retention in the apical membrane is of particular interest because mutations in other proteins involved in ubiquitylation reactions, kelch-like 3 and cullin 3 , are linked to human hypertension $(12,13)$, presumably because they result in membrane NCC accumulation. However, the roles of RING E3 ligases, such as kelch-like 3 , have generally been found to be distinct from those of Hect E3 ligases, such as Nedd4-2 (14); thus, it remains unclear how the roles of the two classes of ubiquitin ligases interact to modulate NCC activity.

The increased abundance of NCC in the Nedd4-2-knockout animals is a mirror image of decreased NCC abundance that results from inducible kidney-specific SGK1 deletion (15). This suggests that Nedd4-2 in the distal convoluted tubule (DCT) may be part of an aldosterone/SGK1/Nedd4-2/
NCC signaling pathway (involving steps i and ii in Figure 1B). As noted, while SGK1 was identified initially as a component of the signaling pathway linking aldosterone to $\mathrm{ENaC}$, it has been increasingly recognized that aldosterone and SGK1 also modulate NCC (16) and that this effect may be physiologically important. What is most impressive in the paper by Ronzaud and colleagues (8) is that NCC levels are increased despite low plasma aldosterone levels; this suggests that Nedd4-2 plays an essential role in aldosterone signaling to NCC. One note of caution, however, is needed: lest we conclude that indeed Nedd4-2 is more important to signal NCC than ENaC, Loffing-Cueni and colleagues (17) reported previously that Nedd4-2 abundance is much lower and less subject to regulation along the NCC-expressing DCT than along the ENaC-expressing connecting tube (CNT). In the current work, we are not told whether dietary salt intake still affects NCC activity.

\section{Conclusions}

Given the above, what can we conclude concerning the role of SGK1 and Nedd42 along the nephron? First, it seems that aldosterone, and probably SGK1, increase 
apical $\mathrm{ENaC}$ abundance largely through signaling mechanisms that do not require Nedd4-2. This conclusion derives not only from the current paper (8), but also from work showing that aldosterone responsiveness of $\mathrm{ENaC}$ in the rectum is increased rather than decreased when Nedd4-2 is deleted (18) and that aldosterone still regulates $\mathrm{ENaC}$ trafficking in the kidney when PY motifs are deleted in vivo (4). One possibility, suggested by the current results, is that Nedd4-2-mediated ubiquitylation primarily accelerates $\mathrm{ENaC}$ degradation within the cell (step iii in Figure 1A) rather than its removal from the membrane (step ii in Figure 1A). Secondly, Nedd4-2 appears to play a physiologically significant and nonredundant role in modulating NCC abundance. That said, it should be pointed out that SGK1 also activates NCC through pathways independently of Nedd4-2 (16) and that other NCC modulators, such as WNK4, may also have large effects on NCC activity. Finally, this work shows again why it is so important to test mechanistic models in vivo. While the results are sometimes confusing and can challenge accepted dogma, their relevance to human health and disease is beyond question. An intriguing clinical implication of the paper by Ronzaud and colleagues (8) is that manipulating Nedd4-2 activity might be a novel approach to treating Gitelman syndrome, an ionic imbalance disease linked to reductions in membrane NCC (19). As Nedd4-2 may inhibit both membrane removal and intracellular deg- radation, its inhibition might increase NCC abundance enough to ameliorate the troubling symptoms of the disease.

\section{Acknowledgments}

The author acknowledges the review and suggestions provided by James McCormick. The author's laboratory is supported by funds from the Department of Veterans Affairs and the NIH (DK 5 RO1 51496 and DK 1 RO1 095841).

Address correspondence to: David H. Ellison, Division of Nephrology and Hypertension, CH12R, Oregon Health and Science University, 3181 SW Sam Jackson Park Road, Portland, Oregon 97239, USA. Phone: 503.494.8490; Fax: 503.494.5330; E-mail: ellisond@ohsu.edu.

1. Staub O, et al. WW domains of Nedd4 bind to the proline-rich PY motifs in the epithelial $\mathrm{Na}^{+}$ channel deleted in Liddle's syndrome. $E M B O J$. 1996;15(10):2371-2380.

2. Rotin D. Role of the UPS in Liddle syndrome. BMC Biochem. 2008;9(suppl 1):S5.

3. Abriel H, et al. Defective regulation of the epithelial $\mathrm{Na}+$ channel by Nedd 4 in Liddle's syndrome. J Clin Invest. 1999;103(5):667-673.

4. Pradervand S, et al. Dysfunction of the epithelial sodium channel expressed in the kidney of a mouse model for Liddle syndrome. J Am Soc Nephrol. 2003;14(9):2219-2228.

5. Ko B, Kamsteeg EJ, Cooke LL, Moddes LN, Deen PM, Hoover RS. RasGRP1 stimulation enhances ubiquitination and endocytosis of the sodiumchloride cotransporter. Am J Physiol Renal Physiol. 2010;299(2):F300-F309.

6. Arroyo JP, et al. Nedd4-2 modulates renal Na+-Clcotransporter via the aldosterone-SGK1-Nedd4-2 pathway. J Am Soc Nephrol. 2011;22(9):1707-1719.
7. McCormick JA, Bhalla V, Pao AC, Pearce D. SGK1: a rapid aldosterone-induced regulator of renal sodium reabsorption. Physiology (Bethesda). 2005;20:134-139.

8. Ronzaud C, et al. Renal tubular NEDD4-2 deficiency causes NCC-mediated salt-dependent hypertension. J Clin Invest. 2013;123(2):657-665.

9. Traykova-Brauch $\mathrm{M}$, et al. An efficient and versatile system for acute and chronic modulation of renal tubular function in transgenic mice. Nat Med. 2008;14(9):979-984.

10. Shi PP, et al. Salt-sensitive hypertension and cardiac hypertrophy in mice deficient in the ubiquitin ligase Nedd4-2. Am J Physiol Renal Physiol. 2008; 295(2):F462-F470.

11. Boase NA, et al. Respiratory distress and perinatal lethality in Nedd4-2-deficient mice. Nat Commun. 2011;2:287.

12. Louis-Dit-Picard H, et al. KLHL3 mutations cause familial hyperkalemic hypertension by impairing ion transport in the distal nephron. Nat Genet. 2012;44(4):456-460.

13. Boyden LM, et al. Mutations in kelch-like 3 and cullin 3 cause hypertension and electrolyte abnormalities. Nature. 2012;482(7383):98-102.

14. Metzger MB, Hristova VA, Weissman AM. HECT and RING finger families of E3 ubiquitin ligases at a glance. J Cell Sci. 2012;125(pt 3):531-537.

15. Faresse $\mathrm{N}$, et al. Inducible kidney-specific Sgk1 knockout mice show a salt-losing phenotype. American journal of physiology. Am J Physiol Renal Physiol. 2012;302(8):F977-F985.

16. Rozansky DJ, et al. Aldosterone mediates activation of the thiazide-sensitive $\mathrm{Na}-\mathrm{Cl}$ cotransporter through an SGK1 and WNK4 signaling pathway. J Clin Invest. 2009;119(9):2601-2612.

17. Loffing-Cueni D, et al. Dietary sodium intake regulates the ubiquitin-protein ligase nedd4-2 in the renal collecting system. J Am Soc Nephrol. 2006;17(5):1264-1274.

18. Bertog $\mathrm{M}$, et al. Aldosterone responsiveness of the epithelial sodium channel $(\mathrm{ENaC})$ in colon is increased in a mouse model for Liddle's syndrome. J Physiol. 2008;586(2):459-475.

19. Kunchaparty S, et al. Defective processing and expression of the thiazide-sensitive $\mathrm{Na}-\mathrm{Cl}$ cotransporter as a cause Gitelman's Syndrome. Am J Physiol Renal Physiol. 1999;277(4 pt 2):F643-F649.

\title{
Multiple functions of a glioblastoma fusion oncogene
}

\author{
Ivan Babic and Paul S. Mischel
}

Ludwig Institute for Cancer Research, UCSD, La Jolla, California, USA.

\begin{abstract}
RNA sequencing facilitates the discovery of novel gene fusions in cancer. In this issue of the JCI, Parker et al. identify an FGFR3-TACC3 fusion oncogene in glioblastoma and demonstrate a novel mechanism of pathogenicity. A miR-99a binding site within the $3^{\prime}$-untranslated region ( 3 'UTR) of FGFR3 is lost, releasing FGFR3 signaling from miR-99a-dependent inhibition and greatly enhancing tumor progression relative to WT FGFR3. These results provide compelling insight into the pathogenicity of a novel fusion oncogene and suggest new therapeutic approaches for a subset of glioblastomas.
\end{abstract}

Conflict of interest: The authors have declared that no conflict of interest exists.

Citation for this article: J Clin Invest. 2013; 123(2):548-551. doi:10.1172/JCI67658.
Genomic technologies are transforming our knowledge about the mutational landscape of cancer. For glioblastoma, the most common and lethal form of adult primary brain cancer, integrated DNA, transcriptional, and epigenetic analyses have identified copy number alterations, mutations, tumor transcriptional and epigenetic subclasses, and potential new drug targets (1-5). Recent progress in next-generation sequencing technologies, including RNA sequencing, provides a powerful new platform adding to this integrated toolkit. Researchers are now able to identify and quantify changes in both coding and noncoding RNA; identify alternative splicing events; and detect expressed mutations, SNPs, gene 